\title{
Special issue on computational intelligence algorithms and applications
}

\author{
Daniel Neagu ${ }^{1}$
}

Published online: 12 July 2016

(C) The Author(s) 2016. This article is published with open access at Springerlink.com

The UK Workshop on Computational Intelligence (UKCI) is the premier UK and Republic of Ireland event for presenting leading research on all aspects of computational intelligence. The 14th Annual Workshop on Computational Intelligence UKCI $2014^{1}$ was organized and hosted from September 8 to 10, 2014 by the Artificial Intelligence Research Group of the University of Bradford, UK, with the support of past UKCI workshop organizers' committee, the network of reviewers and volunteers, and the contribution of authors and keynote speakers. The three keynote talks ${ }^{2}$ delivered by Professor Peter Cowling (University of York's Centre for Complex System Analysis), Mark Barrett (Open Data Lead for Leeds), and Professor Antony Cohn (Director of the Institute for Artificial Intelligence and Biological Systems, University of Leeds), covered cutting edge technologies and developments for the research and industry on Computational Intelligence Research for Impact in the Games Industry, Deriving the Most Value Out of Local Data, and Learning about Activities and Objects from Video, respectively.

The workshop attracted solid interest from prospective authors from all around the world: thirty-two long papers and twelve short papers on recent progress in computational intelligence techniques and applications were selected and presented. From these, authors of twelve best papers, selected by the International Programme Committee and reviewers, were invited for submission of substantially extended versions to be considered for publication in a special issue dedicated to recent advances in computational intelligence algorithms and applications.

\footnotetext{
Daniel Neagu

D.Neagu@bradford.ac.uk
}

1 School of Electrical Engineering and Computer Science, University of Bradford, Bradford, UK
This special issue contains the most recent advances in computational intelligence algorithms and their applications as progressed from the initial scientific contributions, and introduces emerging computational intelligence concepts, algorithms, developments, evidences, and their industry implementations.

Emerging computational intelligence concepts and are covered in six research contributions. In the paper " $F u z z y$ rule weight modification with particle swarm optimization", Tianhua Chen et al. propose an alternative approach using particle swarm optimization in the search of a set of optimal rule weights, entailing high classification accuracy, and demonstrate that the proposed approach can boost classification performance, especially when the size of the initially built rule base is relatively small. Contributions of Lifei Chen et al. in the paper "Kernel-based linear classification on categorical data" extend Naive Bayes, nearest neighbor, and prototype-based classification to classify categorical data and propose two data-driven approaches to the bandwidth selection problem, with one aimed at minimizing the mean squared error of the kernel estimate and the other to attribute weights optimization. Gang Yao and co-authors propose in the paper "Integration of classifier diversity measures for feature selection-based classifier ensemble reduction" a new ensemble subset evaluation method that integrates classifier diversity measures into a novel classifier ensemble reduction framework. The framework converts the ensemble reduction into an optimization problem and uses the harmony search algorithm to find the optimized classifier ensemble. Maktuba Mohid et al. demonstrate for the first time in the paper "Evolution-in-materio: solving computational problems using carbon nanotube-polymer composites" that

\footnotetext{
${ }^{1}$ http://www.computing.brad.ac.uk/ukci2014/.

2 http://www.computing.brad.ac.uk/ukci2014/keynotes.php.
} 
evolution-in-materio methodology can be applied to function optimization and also to the tone discriminator problem. The authors of the paper "Term frequency with average term occurrences for textual information retrieval" (O.A.S. Ibrahim and D. Landa-Silva) introduce a new term weighting scheme that is based on computing the average term occurrences of terms in documents, and it uses a discriminative approach based on the document centroid vector to remove less significant weights from the documents, improving effectiveness and performance in information retrieval with no information on the relevance judgement for the collection. Uzor Chigozirim and co-authors investigate in the paper "Adaptive-mutation compact genetic algorithm for dynamic environments" variants of the recently proposed adaptive-mutation compact genetic algorithm and present a change trend scheme for it so as to boost its performance whenever a change occurs.

Computational intelligence applications and innovative use of such technologies and algorithms are reported and proposed in the remaining six articles, which contribute with high-quality proposals and viewpoints to expand and experiment in domains with high impact to industry and society. Jayne Eaton et al. investigate in the paper "Ant colony optimization with immigrants schemes for the dynamic railway junction rescheduling problem with multiple delays" several different ant colony optimization algorithms applied to the problem of a dynamic train delay scenario with multiple delays: the algorithms resequence the trains at the junction and at the stations: a first step toward expanding the problem to consider a larger area of the railway network. Pritesh Mistry and co-authors demonstrate in the paper titled "Using random forest and decision tree models for a new vehicle prediction approach in computational toxicology" the use of machine learning techniques to process, extract, and build models based on classifiers (decision trees and random forests) that predict which vehicle would be most suited to reduce a drug's toxicity; the authors propose a methodology using an area under the curve (AUC) approach that distinguishes which vehicle provides the best toxicity profile for a drug and builds classification models based on this knowledge using data from the National Institute of Health's (NIH) Developmental Therapeutics Program. Fangyi Li et al. use mean image transformations as a basis for model building and demonstrate, by employing a fuzzy-entropy-based metric, improved ability to model different types of uncertainty in the paper "Handwritten Chinese character recognition using fuzzy image alignment".
Research reported in "Application of permutation genetic algorithm for sequential model building-model validation design of experiments" by M.R. Kianifar et al. is motivated by a complex multivariate engineering problem associated with engine mapping experiments, which require efficient design of experiment (DoE) strategies to minimize expensive testing and is extended with the proposal of algorithms that address issues with non-orthogonal design spaces, that is a common problem in engineering applications. T.D. Sikora and G.D. Magoulas investigate in the paper "Evolutionary approaches to signal decomposition in an application service management system" the potential of population-based metaheuristic algorithms, particularly variants of particle swarm, genetic algorithms, and differential evolution methods, for activity signal deconvolution when the application performance model is a priori unknown. H. Raza et al. present in the paper "Adaptive learning with covariate shift-detection for motor imagery-based brain-computer interface" a covariate shift-detection and self-adaptation methodology, and its application to motor imagery-based brain-computer interfaces; a covariate shift-detection test based on an exponential weighted moving average model is used to detect the covariate shift in the features extracted from motor imagery-based brain responses.

This entire exciting collection of theory and applications of computational intelligence research could not have been possible to be shared with the research community without the active and encouraging support of Professor Vincenzo Loia, the Editor-in-Chief, and Springer's Soft Computing journal team to deliver this Special Issue for presenting the best selection of extended relevant papers from UKCI 2014, and we are grateful to them all. We would like to acknowledge the continuous support of peer-reviewers ${ }^{3}$ and authors who contributed to this special issue. We hope that this collective effort brings to readers a great selection of research contributions that mark the progress and promote scientific excellence in computational intelligence and machine learning theory and applications.

\section{Compliance with ethical standards}

Conflict of interest The author declares he has no conflict of interest.

Open Access This article is distributed under the terms of the Creative Commons Attribution 4.0 International License (http://creativecomm ons.org/licenses/by/4.0/), which permits unrestricted use, distribution, and reproduction in any medium, provided you give appropriate credit to the original author(s) and the source, provide a link to the Creative Commons license, and indicate if changes were made.

\footnotetext{
3 http://www.computing.brad.ac.uk/ukci2014/organising-committee. php.
} 